\title{
El barrio de Arriasa y tres elementos de la aljama judía de Toledo en el siglo XV: la carnicería, la «sinagoga vieja» y el «castillo viejo»
}

Jean Passini*

CNRS, Paris

Mencionado ya en el siglo XIII, el barrio de Arriasa había sido uno de los sectores de la Judería de Toledo más difíciles de localizar. Menciones en contratos conservados de arrendamiento de casas desde fines del siglo XIII y hasta la segunda mitad del siglo XVIII, y las vicisitudes sufridas por las propiedades inmuebles de la aljama judía desde fines del siglo XV han permitido situar la carnicería, la «sinagoga vieja» y el «castillo viejo». De esta manera se delimita claramente el barrio de Arriasa, que se distingue del barrio del Degolladero, aclarando además las referencias a la «sinagoga vieja» y al «castillo viejo».

Palabras Clave: Judería; Toledo; Arriasa; bienes comunes; «sinagoga vieja»; «castillo viejo».

The Arriasa Neighborhood and Three Elements of the $15^{\text {Th }}$ Century Jewish Community of Toledo: The Butcher's Stalls, the "Old Synagogue" and the "Old CAstle".- - Already mentioned in $13^{\text {th }}$ century written sources, the Arriasa neighborhood was an area within the Toledan Jewish quarter that still remained to be identified and demarcated. Documentary evidence -tenancy agreement charters dated back from the $13^{\text {th }}$ until the $18^{\text {th }}$ centuries-, as well as information on the vicissitudes experienced by the Jewish common properties from the late $15^{\text {th }}$ century on, have been used to locate the butcher's stalls, the "old synagogue" and the "old castle" on a street plan. Thus, the Arriasa neighborhood has been clearly delimited.

KEYwords: Jewish Quarter; Toledo; Arriasa; Jewish Common Properties; "Old Synagogue;" "Old Castle."

La mayor dificultad que se experimenta cuando intentamos investigar la historia de la judería de Toledo y más concretamente su topografía urbana se debe a la extrema escasez tanto de testimonios escritos, casi inexistentes en lo que se refiere a su época más floreciente en los siglos XI-XII, como de construcciones pertenecientes a esta época. Cuando los judíos tuvieron que dejar sus casas, primero a fines del siglo XIV, a raíz de los violentos tumultos de 1391 y, después, ya de manera masiva, en 1492, el barrio judío al sur del de Santo Tomé quedó en un estado de semiabandono antes de ser sometido a intensos trabajos de transformación, desde la segunda mitad del siglo XIX. La inexistencia de vestigios fiables en

\footnotetext{
*jpassini@free.fr
} 
su suelo, si exceptuamos dos sinagogas edificadas en el siglo XIV, y el hecho de que se instalaran, conventos en el área de lo que había sido antiguamente la judería conduce, a una visión a veces errónea de ese espacio de Toledo.

Fieles al método que hemos desarrollado y perfeccionado en nuestro estudio del barrio sur de la catedral ${ }^{1}$, nos hemos dedicados, a un análisis detallado de textos, no todos conocidos, de numerosos autores que se dedicaron al estudio de la judería de Toledo $^{2}$, y a la búsqueda de algunos puntos que nos puedan servir de referentes seguros en el suelo, analizando a diferentes niveles el conjunto de las construcciones actuales. En el artículo que sigue presentamos los primeros resultados de nuestros trabajos acerca de uno de los componentes de la judería en los siglos XIII y XIV, el barrio de Arriasa, y de otros tres elementos de los bienes de la comunidad judía cuya existencia está documentada a fines del siglo XV: la antigua sinagoga, la «carnicería de los judíos» y el «castillo viejo», elementos cuya situación en la judería no estaba hasta hoy definida con exactitud.

\section{BARRIO DE ARRIASA}

Ya hemos señalado que los textos que pueden aclararnos algo acerca de la estructura de la judería son poco numerosos; no obstante, no hay duda de que se componía de varios barrios de distinta extensión ${ }^{3}$, entre ellos el de Arriasa, que debía de existir desde bastante antes del último decenio del siglo XIII. En 1292, en efecto, el monasterio de San Clemente compra tres casas - «una almazría, un sótano y seis tiendas»- situadas juntas «en el Arrabal de los Judíos, en la calle

${ }^{1}$ J. Passini, J. P. Molénat, Toledo a finales de la Edad Media. I. El barrio de los Cánonigos (Toledo 1995); J. PAssinI, Casas y casas principales urbanas. El espacio doméstico de Toledo a fines de la Edad Media (Toledo 2004).

${ }^{2}$ Sólo citaremos a los principales autores que iniciaron este camino: F. CANTERA Burgos, Sinagogas españolas (Madrid 1955, reimpr. 1984); IDEM, Sinagogas de Toledo, Segovia y Córdoba (Madrid 1973); P. León Tello, Judios de Toledo (Madrid 1979); J. Porres Martín-Cleto, «La judería de Toledo después de la expulsión», en La expulsión de los Judíos de España. II Curso de Cultura hispanojudía y sefardí. Toledo, 16-19 septiembre 1992 (Toledo 1993), págs. 26-46.

3 Ver J. Porres Martín-Cleto, «Algunas precisiones sobre las juderías toledanas», Anales Toledanos 14 (1983), 37-61; pág. 41: «[...] la Judería en sí, o el barrio que como tal se conocía en los siglos anteriores a 1492, no era una agrupación uniforme de viviendas con varias sinagogas entre ellas. Dentro del muro había barrios diferentes [...] Las más importantes de estas agrupaciones se citan con mayor claridad en los siglos XIV-XV, como son de Alacava, Caleros, Santo Tomé, el Degolladero de los Judíos, Hamanzeite, Cosperos, postigo del Fierro, calle o barrio de Arriaza, etc., como barrios conocidos y diferenciados, distintos de la Judería en sí». 
del barrio Arriasa, en la ciudad de Toledo» ${ }^{4}$. Bajo la tercera casa, la que estaba más abajo, haciendo esquina, había seis tiendas. Tres daban a la plaza «donde están las tiendas de los carniceros», y las otras tres hacían frente a las tiendas que se apoyaban contra el muro del castillo, en la calle «que fue del alguacil almojarife Abuibrahim» (fig. 1). Las tres casas seguirán siendo propiedad del monasterio hasta la segunda mitad del siglo XVIII ${ }^{5}$.

El barrio de Arriasa incluía, por lo tanto, al menos la calle del barrio de Arriasa ; otra calle, de actividad comercial, perpendicular a ésta; y por detrás de las tres casas descritas más arriba, la plaza de los carniceros. El barrio se acababa en el castillo, al que posiblemente rodeaba, pero no incluía. Un castillo cuya razón de ser consistía en proteger los barrios vecinos y cobijar a los habitantes de la judería en caso de ataque, como pasó en $1355^{6}$, y que era potencialmente un barrio en sí mismo. Este barrio mantenía su nombre de Arriasa, o de Harrat Arrieça, a fines del siglo XIV, como vemos en documentos notariales de $1353^{7}$ y $1396{ }^{8}$. Un siglo más tarde, tras la expulsión de judíos y musulmanes, este

4 A. González Palencia, Los Mozárabes de Toledo en los siglos XII y XIII (Madrid 1929), doc. 710 .

${ }^{5}$ Las casas, adquiridas en 1292, se localizan a lo largo del tiempo gracias a los contratos de alquiler publicados por León Tello, Judíos de Toledo, t. II. En 1353 la superiora de San Clemente da a censo «unas casas en la judería de Toledo en la calle que dicen de Harrat Arrieça, con su almaçería [...] linda con casas del monasterio [...] e la bodega» (doc. 490). En 1365, la superiora de San Clemente alquila a don Mosé, hijo de Abrahem Nahamán, judío de Toledo, «dos pares de casas y almacería, en la judería, donde moraba el mismo don Mosé, y tres tiendas abiertas para labrar de herrería [...] lindan con casas del convento» (doc. 544). En 1484, «doña Cinhá [...] vende [...] unas casas en Toledo, en la colación de Santo Tomé, en la judería de esta ciudad, con su tienda, bóveda y cámaras que lindan [...] y afrontan el castillo de la judería y por delante la calle pública real» (doc. 1484). A la casa que hacía esquina corresponde, en el siglo XVII, la casa $\mathrm{n}^{\circ} 12$ del repertorio de los bienes de San Clemente, redactado en 1770.

${ }^{6}$ Véase P. López de Ayala, Crónica del Rey don Pedro, cap. 6 y 7 (año 6), ed. BAE, t. 66, págs. 462-463, cit. J.-P. MoléNAT, «Quartiers et communautés à Tolède (XII ${ }^{\mathrm{e}}-\mathrm{XV}^{\mathrm{e}}$ siècles)», En la España Medieval 12 (1989), 164-189, pág. 171, nota 35.

${ }^{7}$ LeÓn Tello, Judíos de Toledo, t. II, doc. 490; la superiora de San Clemente da en 1353 a censo «unas casas en la judería de Toledo en la calle que dicen de Harrat Arrieça, con su almaçería [...] linda con casas del monasterio [...] e la bodega».

8 Archivo del monasterio de Santo Domingo el Real [= ASDR], L 3-16, «Asiento de todas las posesiones del monasterio de Santo Domingo el Real, comenzado el año 1507» [= Becerro 1507], transcrito por Amalia Yuste. Este documento copia el acta de venta a sor Marina Rodríguez de Valladolid «de unas casas con las almaçerías que se tienen con las dichas casas aquí en Toledo en la Judería en el adarve de Harretarrieça por 2.000 mrs. [...] fecha a 26 días de junio año de 1396». En este texto se denomina «adarve de Harretarrieça» a la calle de «barrio Arriasa». 


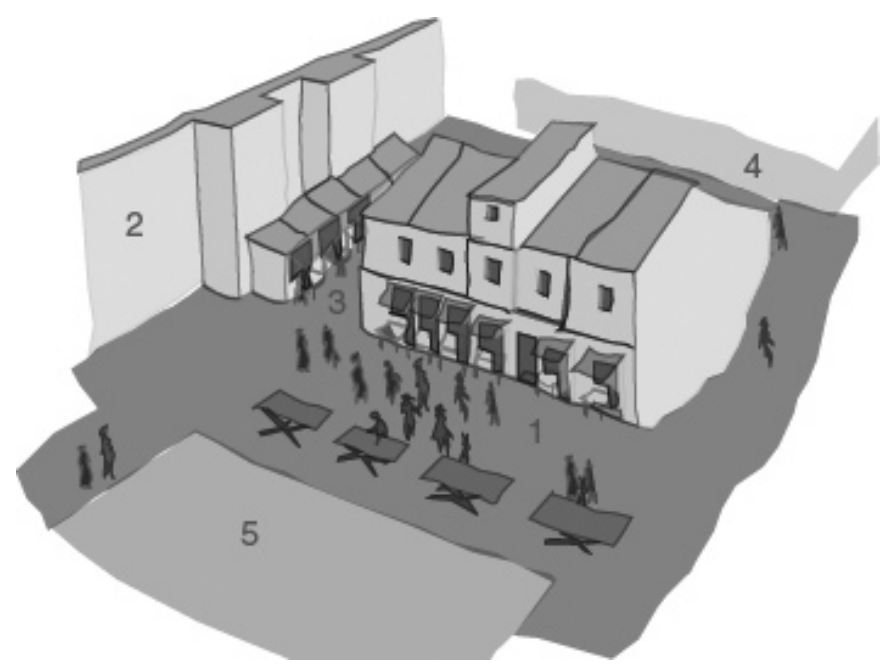

Fig. 1. Barrio de Arriasa: las casas compradas por el convento de San Clemente en 1292, en la esquina de la carnicería y enfrente del castillo.

1. Plaza de los Carniceros. 2. Castillo viejo. 3. Calle del alguacil almojarife Abuibrahim.

4. Calle del barrio de Arriasa. 5. Sinagoga vieja.

nombre ya no se utilizará ${ }^{9}$; lo sustituirá el binomio Barrio Nuevo, que designa todo el conjunto de la judería al sur de Santo Tomé.

Ya hemos dotado de un contenido al barrio de Arriasa a fines del siglo XIII; intentaremos, en lo que sigue, localizar los puntos de referencia principales del barrio, a saber, la plaza de los carniceros y el castillo viejo de los judíos.

\section{CARNICERÍA DE LOS JUDÍOS}

Acabamos de ver que a fines del siglo XIII existía en la judería, más precisamente en el barrio de Arriasa, enfrente del castillo, una plaza donde estaban las tiendas de los carniceros. La expresión «plaza de los carniceros» no aparece en ningún otro documento relativo a bienes del «arrabal de los judíos»; sin embargo, sí se menciona la «carnicería de los judíos». ¿Podemos admitir que esta

${ }^{9}$ ASDR, L 3-16 (Becerro 1507). El documento registra la entrega a censo en 1492 de una casa adquirida por el monasterio en 1396. Se explica que la casa se encuentra en un adarve que «se solía llamar antiguamente de Harratariça». 
última entidad incluye la plaza de los carniceros? Nos proponemos responder a este interrogante centrándonos en la citada carnicería, lo que consolidará nuestra reflexión sobre el barrio de Arriasa.

En varios documentos notariales de los siglos XIII y XIV se dice que el cabildo de la catedral, determinados alguaciles y otros personajes de la ciudad reciben tributos de la carnicería de los judíos ${ }^{10}$. Hasta el momento no disponemos de ningún documento anterior a 1492 que nos permita precisar el lugar en donde se levantaba la carnicería. Promulgado en este año el decreto de expulsión, algunos personajes importantes de la ciudad, privados de los tributos que recibían de las casas sujetas a censo, abandonadas por sus moradores judíos, piden una compensación a los reyes. Entre estas peticiones figura la del regidor Fernando Dávalos, su hermano Alonso y Fernán Suárez, a la que responden los Reyes Católicos en marzo de 1494, ordenando a su consejero y corregidor de Toledo que les conceda el derecho de vender «la sinoga vieja que los judios tenian en la dicha ciudad, cerca de las carnecerias de los judios con todas las cosas a ella anexas e pertenescientes» ${ }^{11}$, como indemnización por las pérdidas sufridas.

Tres años más tarde, en enero de 1497, Fernán Suárez y los hermanos Dávalos dan a censo al comerciante Diego de Villareal y a su mujer Elvira López la sinagoga que linda con las casas de Fernán Álvarez de Toledo, el muro que corona el rodadero del Tajo y «la carniceria que antes era de los judios», y con el corral de Salvador Valverde ${ }^{12}$. Diego de Villareal se compromete a hacer algunos arreglos y algunas habitaciones nuevas durante los tres primeros años. A partir de este momento, no sabemos con certeza qué ocurre con este vasto inmueble; sólo podemos seguir la pista de algunos elementos de la vieja sinagoga a través de los pleitos entre la familia Dávalos y los frailes del monasterio de San Agustín ${ }^{13}$. En 1511, en efecto, los tres sobrinos de Alonso Dávalos se reparten algunos bienes de éste a fin de dotar la fundación que su tío dejó al convento de San Agustín en la capilla del condestable Ruy López Dávalos ${ }^{14}$, y que consistían, entre otros, en

${ }^{10}$ León Tello, Judíos de Toledo, t. II, docs. 163 (1366), 197 (1260), 427 (1345) y 477 (1351).

${ }^{11}$ León Tello, Judíos de Toledo, t. I, doc. 93, págs. 609-613.

12 Documento del 7 de enero de 1497, publ. León Tello, Judíos de Toledo, t. I, págs. 359-360. El censo asciende a $5.000 \mathrm{mrs}$. y cinco pares de gallinas.

${ }^{13}$ Los frailes del convento de San Agustín tenían a su cargo la capilla de San Esteban.

${ }^{14}$ Archivo Histórico Nacional [= AHN], Clero, Papeles, leg. 7164 (Toledo, capilla de San Esteban). Tributo sobre casas en Santo Tomé en el adarve, y otras tres casas en dicho adarve, casas en la sinagoga vieja (1511). Partición judicial que se hizo entre don Fernando Dávalos y Pedro 
dos tributos sobre otras tantas casas que estaban juntas: uno, por importe de 500 mrs., sobre las casas de Gutierre de Palma que lindaban con la «carniceria que fue de los judios»; el otro, de 670 mrs., sobre las del sastre Gonzalo Ruiz ${ }^{15}$. La lectura del libro de las propiedades del monasterio de San Agustín, realizado en 1577, concretamente del capítulo relativo a la capilla de San Esteban ${ }^{16}$, nos muestra que la casa «que era de Gutierre de Palma», llamada en este mismo párrafo «parte de la sinoga», pasa en 1539 a los herederos de Gonzalo de Carmona. La otra casa, ocupada anteriormente por Gonzalo Ruiz y que era parte de «las casas de la dicha sinoga», pasará a Alonso de Ávila «por su sobrino, hijo de Francisco Ruiz», y después a «Luysa de San Juan, su mujer». A partir de este punto carecemos de noticias sobre la casa de Gonzalo Ruiz. Por el contrario, tenemos documentos del siglo XVI que nos informan sobre el devenir de las casas de Gutierre de Palma, que pasaron a la familia de Gonzalo de Carmona. En 1539, Gonzalo de Carmona y Leonor Álvarez venden al canónigo Cristóbal Navarro un tributo de 1.500 mrs. sobre la casa principal que tenían en la parroquia de Santo Tomé ${ }^{17}$, que lindaba por un lado con la de Juan Gómez Ávila y Beatriz de Carmona, por otro con la de los herederos de Gonzalo Ruiz, y por un tercero con la casa del «regidor Juan Niño» y «la cerca de la ciudad» ${ }^{18}$. En 1584, tras un proceso judicial ${ }^{19}$, esta misma «casa de la sinoga» se vende a Sebastián de Mora, que un año más tarde acepta el tributo de 1.500 mrs. a favor del Capítulo de Curas y Beneficiados sobre la casa que posee «a la xinoga vieja». Este mismo tributo lo aceptan en 1598 la viuda de Sebastián Mora y, a fines del XVII, Martín Ramírez.

Conviene que retengamos de todo lo anterior que apoyada en la carnicería de los judíos, entre ésta y la muralla que se cierne sobre el Tajo, se encontraba la «sinagoga vieja», de la que sabemos además que fue intensamente dañada durante los tumultos del verano de $1391{ }^{20}$.

Vélez de Guevara y Aldonza Carrillo. El resumen de estos documentos figura en León Tello, Judios de Toledo, t. II, pág. 608, doc. n 1737.

${ }^{15}$ Los procesos siguientes serán, en lo esencial, repetición del juicio de 1511. En 1523, tras la ejecución de Fernando Dávalos, los herederos de don Alonso Dávalos son condenados a aportar 3.666 mrs. y 4 coronas para dotar la fundación de Fernando Dávalos en la capilla de San Agustín.

16 AHN, Clero, Libros 14951 (Libro de posesiones del convento de San Agustín).

17 Casa comprada anteriormente a Gutierre de Guadalupe, «mercador».

18 Archivo Histórico Provincial de Toledo [= AHPT], H-1924/6. Tributo de 1.500 mrs. pagado al Capítulo de Curas y Beneficiados.

19 AHPT, H-1924/6.

${ }^{20}$ Debido a su importancia, esta sinagoga («templo viejo») es la segunda mencionada por Albené en el poema compuesto tras el saqueo de la comunidad judía: «Y el Templo Viejo / Era como mitad de granada / [...] / Extranjeros llegáronse a sus puertas / Y arrojaron de allí sus 


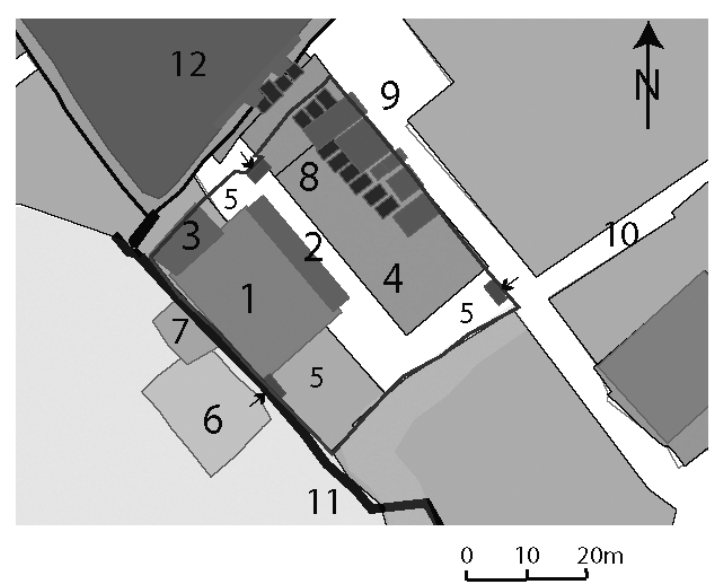

Fig. 2. Plano hipotético de la «carnicería de los judíos» en el siglo XV.

1. Sinagoga vieja. 2. Portal de los carniceros. 3. Hospital de la aljama. 4. Carnicería de los Judíos. 5. Entradas de las carnicerías de los judíos. 6. Corral conocido como «amarradero de las vacas». 7. Huerta pequeña y covachuelas. 8. Tiendas y sótanos que abren a la plaza de los carniceros. 9. Casa encima de las tiendas que abre a la calle del Barrio de Arriasa. 10. Calle de la Judería, actual calle de San Juan de Dios. 11. Muro de la ciudad. 12. Castillo de los judíos.

En el inventario de los bienes de la aljama judía de 1494 vemos que la carnicería de los judíos comprendía, al menos, seis casas que estaban juntas, un corral, varios portales, un hospital, un solar entre dos torres y, además, una puerta principal ${ }^{21}$. La lista de los bienes que quedaban por vender en 1502 aporta mayor precisión sobre las relaciones espaciales entre la sinagoga, la carnicería y un corral exterior a ésta (fig. 2). Se pasaba de la «sinagoga vieja» a la carnicería por un postigo, junto al cual había un corral conocido como «amarradero de las vacas», donde los judíos sacrificaban los animales destinados al consumo, un pequeño huerto y algunas cuevas pequeñas ${ }^{22}$.

libros / Y colocaron un ídolo entre sus ruinas», cit. CANTERA Burgos, Sinagogas españolas, págs. 35-55.

${ }^{21}$ Publ. León Tello, Judíos de Toledo, t. I, doc. 97, págs. 616-620. Entre los bienes comunes de la aljama figuran «seis pares de casas que son juntas unas con otras en una hasera que son sobre las dichas carnecerias que fueron de los dichos judios e con las dichas carnecerias con cierta parte del corral e con la entrada de la puente mayor de las dichas carnecerias e con los portales e carnecerias e tajona e con una casa questa debaxo de las dichas casas que fueron ospital de la dicha aljama e con un solar de entre dos torres junto con la dicha puerta que tenia».

${ }^{22}$ Archivo General de Simancas [= AGS], RGS, CC Cédulas, libro 6, doc. 115, f. o 25v (1502): «[...] un corral donde los judios mataban los animales para abastecer sus carnicerias conocido 
Podemos precisar la situación de la carnicería de los judíos si seguimos la pista de ciertos elementos que eran parte integrante de ella. En 1498, Fernán Suárez posee en «la carneçeria que solia ser de los judios» un suelo y «una sala, que linda con las paredes de la casa que era xinoga, un portal e un corredor», que da a censo a Lorenzo de Guadalupe y a Isabel de Limpias por $1.240 \mathrm{mrs} .{ }^{23}$. Exige a los nuevos ocupantes que hagan «una puerta de alfacen» ${ }^{24}$, a fin de preservar su propia intimidad en los dos palacios que reserva para sí en la misma casa. En 1501, Isabel de Limpias vende esta casa, que está en el interior de la carnicería de los judíos, adosada a los palacios de Fernán Suárez, la casa de Gómez de Carmona, la de Luis Francés, un corral, la casa de Bartolomé Sánchez y la calle real, a Gonzalo de Toledo y a Catalina Álvarez. En 1516, el hijo de Fernando Suárez vende el tributo anual de $1.240 \mathrm{mrs}$. que grava la casa a Lucas de la Peña, canónigo de la catedral ${ }^{25}$.

Por otra parte, Fernando Suárez era dueño de la casa que ocupaba Bartolomé Sánchez, situada en barrio nuevo, en la antigua carnicería. En $1502{ }^{26}$, la casa es gravada con un tributo anual de 1.000 mrs., tributo que vende en 1506 a las «beatas de la contadora» ${ }^{27}$. La casa de Bartolomé Sánchez linda con las de Gómez de Carmona, con otras casas «que solia ser de la carniceria que son unos palacios de Fernan Suarez y su mujer», y con la calle real. Tiene además un «sotano debajo que manda por la calle». El

como el amarradero de las vacas, un huerto pequeño situado a las espaldas de dicho corral, unas covachuelas junto a este que solian alquilarse [...] todo ello lindaba con la sinagoga mayor, la carniceria de los judios y el postigo que estaba entre ambas $[\ldots]$ ».

${ }^{23}$ Archivo Catedral de Toledo, Obra y Fábrica [= ACT, OF], libro 83 (1498): «[...] Yo, Fernan Suarez, criado del rey y la Reyna, doy a vos, Lorenzo Guadalupe y Isabel de Limpias, [...] un suelo en la carniceria que solia ser de los judios en la collacion de Santo Tome segun y manera que por nosotros que esta. Una sala esta dentro en el mismo suelo y un portal que esta debajo de la dicha sala segun que es el compad de la dicha sala y tres palmos menos que alindan la dicha sala con los paredes de la casa que era xinoga y que por el dicho suelo vos el dicho Lorenzo de Guadalupe [...] fagades una puerta de alfacen por donde vosotros y los moradores que mora y moraron en lo otro que queda a mi, el dicho Ferran Suarez, que he de dar a tributo puedan entrar y salir cada uno, tenga entrada y salida y asimismo queda a mi, el dicho Ferran Suarez, a para mi dentro el dicho suelo y sala que suso dicho es, vos doy apodero [...]».

${ }^{24}$ Puerta con arco entre el zaguán y el patio.

25 ACT, OF, libro 83 (1516).

${ }^{26}$ AHN, Clero, Libro 15315.

27 AHN, Clero, Libro 15615 ( $\sin$ f. $^{\circ}$ ). Con el tiempo, las «beatas de la contadora» serían las monjas del convento de San Antonio. 
censo sobre la casa, a favor de las Beatas, es aceptado en 1529 por la hija de Bartolomé Sánchez, y más adelante por Juan de Perejón de Morgáez, que vende la casa en 1573 al capellán del convento de San Antonio de Padua ${ }^{28}$. En esta fecha se describe el inmueble lindando con la casa de Juan Niño, con «las casas hondas que llaman de la synoga» y con la calle real. A partir de ahora, el nombre sinagoga (o synoga) será el único que siga apareciendo en los documentos, a veces ligado al binomio «casas hondas», expresión esta última inusitada hasta este momento en las aceptaciones de tributos, pero que encontramos en el censo de habitantes de 1561 aplicada a un entorno concreto en la parroquia de Santo Tomé, entre «el miradero» y «la calle de San Benito» ${ }^{29}$. La «casa honda», llamada «synoga vieja» en 1573, será asociada en 1652 a la expresión «sumidero de el agua al barrio nuevo» ${ }^{30}$, como dice la nota escrita en la primera página del libro que compendia las aceptaciones de tributos y las ventas sucesivas de la casa «que solia ser las carnicerias de los judios que son dos palacios [...]» ${ }^{31}$ : «casa a la collacion de Santo Tome junto al çumidero de el agua a barrio nuevo» ${ }^{32}$. Otra casa, que figura en la lista de bienes de la aljama redactada en 1499, aparece ubicada «en el callejoncillo de las casas de Juan Niño» en el siglo XVI, y en la segunda mitad del XVII se describe enfrente del «sumidero de agua» ${ }^{33}$.

${ }^{28}$ En 1573, Valentina de Cuéllar, superiora del monasterio de San Antonio de Padua, compra a Juan de Perejón de Morgáez «unas casas en esta ciudad de Toledo, en la colacion de Santo Tome, que alindan por una parte con casa de Juan Niño, y por la otra con las casas hondas que llaman de la synoga y por delante la calle real».

${ }^{29}$ Este censo, publicado por L. Martz y J. Porres Martín-Cleto, Toledo y los toledanos en 1561 (Toledo 1974), pág. 183, cuenta cincuenta habitantes en la manzana «Casa honda».

${ }^{30}$ Tal como se precisa en la nota de la primera página del libro que compendia las aceptaciones de censos y las ventas sucesivas de la casa «que solia ser las carnicerias de los judios que son dos palacios [...] casa a la collacion de Santo Tome junto al çumidero de el agua a barrio nuevo». El «çumidero de el agua» se ubica en «barrio nuevo» en diversos documentos del siglo XVII. Ver, por ejemplo, el documento de marzo de 1672 del Archivo Municipal de Toledo, que menciona «un muro que amenaza de ruinas junto al sumidero de agua a barrio nuevo [...] junto con el cobertizo de la cruz».

${ }^{31}$ Casa del convento de San Antonio de Padua.

${ }^{32}$ AHN, Clero, Libro 15315. Nota de una escritura del siglo XVII. La primera página de este libro la ocupa el documento de aceptación de un tributo de $1.000 \mathrm{mrs}$. y dos gallinas por Valentín de Cuellar sobre la casa «que linda por una parte con casa de Juan Niño, por la otra con casa honda que llaman de la synoga».

33 AHPT, H-1924/2. El convento de Santa Ana adquiere la casa en 1652. Los documentos de venta permiten seguir las sucesivas aceptaciones del tributo de $300 \mathrm{mrs}$. a favor de Curas y 
El «sumidero de agua» no aparece en las descripciones de casas más que a partir del siglo XVII. Entre los poquísimos documentos que lo mencionan citaremos uno de 1646 del monasterio de Santo Domingo el Real, relativo a una casa de la «calle del Horno», «bajando del Horno de la Oliva para ir al Sumidero de Agua en una casa que hacia esquina antes de llegar a Corpus Christi» ${ }^{34}$. El sumidero de agua se encuentra por lo tanto al final de la calle que baja desde "el horno de la Oliva", por el hospital del Corpus Christi (actual calle de San Juan de Dios), calle que era, y es, vía natural de evacuación de las aguas de lluvia.

El análisis precedente aporta un principio de solución al problema de la localización de la carnicería, precisando la situación de tres lados de la manzana

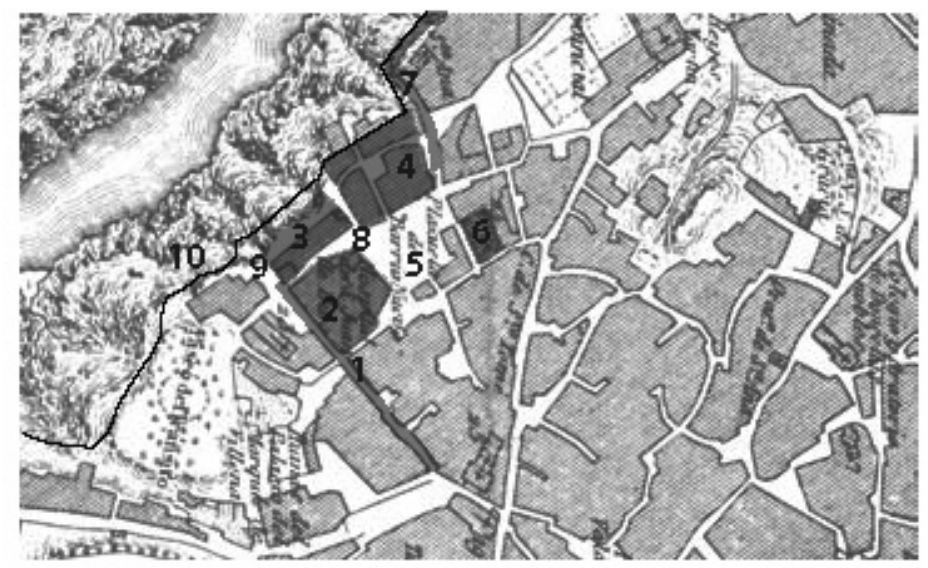

Fig. 3. Plan de Coello, 1858: la calle entre el «castillo viejo» y la carnicería de los judíos.

1. Calle del Horno de la Oliva. 2. Hospital de San Juan de Dios. 3. Manzana de la sinagoga vieja y de la carnicería de los judíos. 4. Manzana del Castillo viejo de los judíos. 5. Plaza del barrio Nuevo. 6. Iglesia de Santa María la Blanca. 7. Cuesta. 8. Calle entre el Castillo viejo y la carnicería. 9. El sumidero de agua. 10. El muro de la ciudad.

Beneficiados que gravaba la casa en $1516,1574,1595,1618$ y 1652 . Esta casa figura en la larga lista de los bienes de la aljama de Toledo redactada en octubre de 1494, León Tello, Judíos de Toledo, t. I, pág. 617. Leonor de Ribera, monja de Santo Domingo el Real, compra en 1494 el tributo de $300 \mathrm{mrs}$. y lo da a Curas y Beneficiados a cambio de una capellanía en favor de su hermano Vasco de Ribera, obispo de Coria. Una nota posterior precisa que «estas casas son en el callejoncillo de las casas de Juan Niño [...] en las casas frontero de las de Francisco de Torres».

${ }^{34}$ ASDR, L 3-16 (Becerro 1507): una casa del monasterio de Santo Domingo el Real , citada en 1507 «a barrio nuevo solia ser juderia», se menciona en 1646 (Libro 3, Becerro) «bajando del Horno de la Oliva para ir al Sumidero de Agua en una casa que hacia esquina antes de llegar a Corpus Christi». 
de la carnicería de los judíos, uno frente a la muralla de la ciudad, otro en la parte final (actual calle Victorio Macho) de la futura calle del Horno de la Oliva (actual calle de San Juan de Dios) hacia el río, el tercero en la calle donde también estaba el «castillo viejo» ${ }^{35}$ (fig. 3). Podemos igualmente concluir de este análisis las dos observaciones siguientes: en primer lugar, la carnicería era un espacio cerrado, que tenía al menos una puerta principal. Además, la «synagoga vieja» fue reemplazada en el siglo XVI por una casa que se conocía como «casa honda» ${ }^{36}$, inmediata al «sumidero de agua» (fig. 4). La vista axonométrica de Arroyo Palomeque ${ }^{37}$ muestra claramente la manzana de la «casa honda» (antiguamente «sinagoga vieja»), entre la muralla de la ciudad y la calle del Horno (o de la Oliva). También muestra la vía de evacuación de las aguas al final de la calle del Horno, a la altura del «sumidero de agua». Ahora nos falta localizar el «castillo de los judíos», el llamado «castillo viejo».

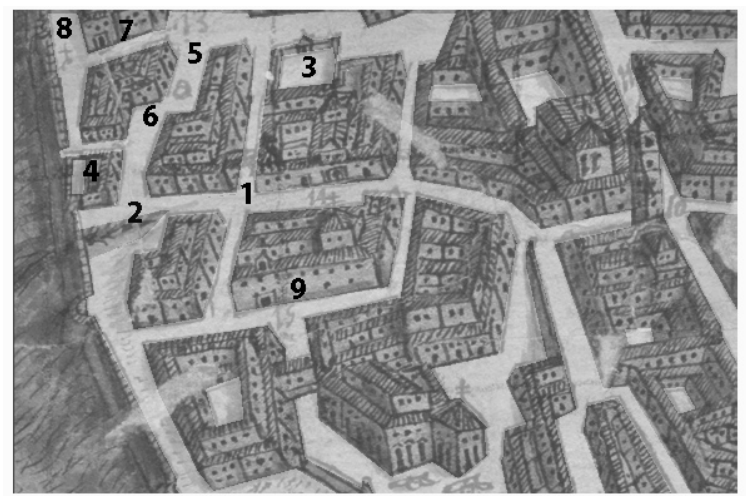

Fig. 4. Plano axonométrico de Arroyo Palomeque: la «casa honda», antiguamente «sinagoga vieja», y el «sumidero de agua».

1. Calle del horno, actual San Juan de Dios. 2. Sumidero de agua. 3. Hospital de San Juan de Dios (antiguamente Corpus Christi). 4. Manzana de la «casa honda» junto a la muralla de la ciudad. 5. Castillo viejo. 6. Pozo del Castillo. 7. Monjas de Sta. Ana. 8. Cuesta. 9. Iglesia de San Benito.

${ }^{35}$ La calle entre el solar del antiguo castillo de los judíos y el de la carnicería figura en el plano de Coello (1858).

${ }^{36}$ La vista axonométrica de 1718-21 de Arroyo Palomeque muestra la manzana de la casa honda, entre la muralla y la calle del horno (o de la Oliva). También destaca la vía de evacuación de aguas al final de la calle del Horno, a la altura del «sumidero de agua».

37 Ver J. Porres Martín-Cleto, R. J. del Cerro Malagón, J. L. Isabel Sánchez, Panorámica de Toledo de Arroyo Palomeque (Toledo 1992). Los autores consideran que la axonometría se realizó entre 1718 y 1721. 
Un pequeño número de documentos lo menciona antes del siglo XV ${ }^{38}$. El «castillo de los judíos» figura en 1163 en la escritura de un préstamo concedido al judío Isaac ben Abuyusef, que pone como garantía la mitad de su casa «en el castillo de los judíos, sobre el río Tajo» ${ }^{39}$. Un siglo después, se explicita la existencia de una calle que va de la puerta del castillo viejo de la judería a la del castillo nuevo ${ }^{40}$. En 1492 se vuelve a mencionar en un documento de aceptación de tributo sobre una casa cercana al castillo que linda con ciertas casas y con la sinagoga de Santa María la Blanca y la «calle real» ${ }^{41}$.

El «castillo viejo» figura en la lista de los bienes de la aljama toledana de $1492{ }^{42}$ : el castillo limitaba con la carnicería y con la cuesta que baja al Tajo; una de sus torres se alzaba junto a la puerta de la carnicería y las «calles públicas». En esta fecha ya no quedaba de él más que un solar y una torre ${ }^{43}$. En 1496, viendo que todo ese espacio estaba «todo fecho muladares e syn provecho», les parece a los funcionarios que lo inspeccionan por orden de los reyes «que en fasello casas se farya barryo poblado, e quedava calle tan ancha y mas que ninguna de la dicha çibdad» ${ }^{44}$. El solar que antaño ocupó el castillo

38 Más numerosos son los que indican la existencia de casas apoyadas sobre castillo, por ejemplo en 1483 «unas casas a la collacion de Santo Tome, en la juderia con tienda boveda [...] linda casa de los herederos de Jacob Abengato [...] y afrentaba con el castillo de la Juderia y por delante la calle real publica [...]», AHPT, H-1339/1, casas varias (1491-1493).

39 León Tello, Judios de Toledo, t. II, doc. 897. MolÉnAt, «Quartiers et communautés à Tolède», págs. 163-189; Porres Martín-Cleto, «La judería de Toledo después de la expulsión», págs. 36-37.

40 En un documento publicado por González Palencia, Los mozárabes de Toledo, $\mathrm{n}^{\circ} 1135$ (1270 noviembre), que registra la venta de una casa en el barrio (arrabal) de los judíos, «[...] en la calle que era adarve [...] por su fondo comunica la dicha calle con la via que se dirige de la puerta de nuestro castillo nuevo a la puerta del castillo viejo» (trad. González Palencia); cit. MoLÉnAT, «Quartiers et communautés à Tolède», pág. 173.

41 AHPT, H-1141/14.

${ }^{42}$ León Tello, Judíos de Toledo, t. I, doc. 97, págs. 616-620: «Yten, el solar del castillo que fue propio de la dicha aljama [...] lindero de la una parte las carnicerias e de la otra parte la cuesta que desciende del dicho castillo al rio, e de la otra parte una torre del dicho castillo que esta junto con la puerta de la dicha carniceria e las calles publicas».

${ }^{43}$ León Tello, Judíos de Toledo, t. I, doc. 97, pág. 619: «El solar del castillo viejo... lindero de la una parte las carnicerias e de la otra parte la cuesta que desciende del dicho castillo al rio, e de la otra parte una torre del dicho castillo que esta junto con la puerta de la dicha carniceria e las calles publicas».

44 AGS, RGS, 25-X-1496, f. 58, cit. O. LóPEZ, Violencia urbana y paz regia: el fin de la Época Medieval en Toledo (1465-1522), tesis doctoral inédita (Toledo, Universidad de CastillaLa Mancha, 2007). 
se divide en parcelas y sobre estos lotes se hicieron casas en el primer cuarto del siglo XVI (fig. 5) ${ }^{45}$.

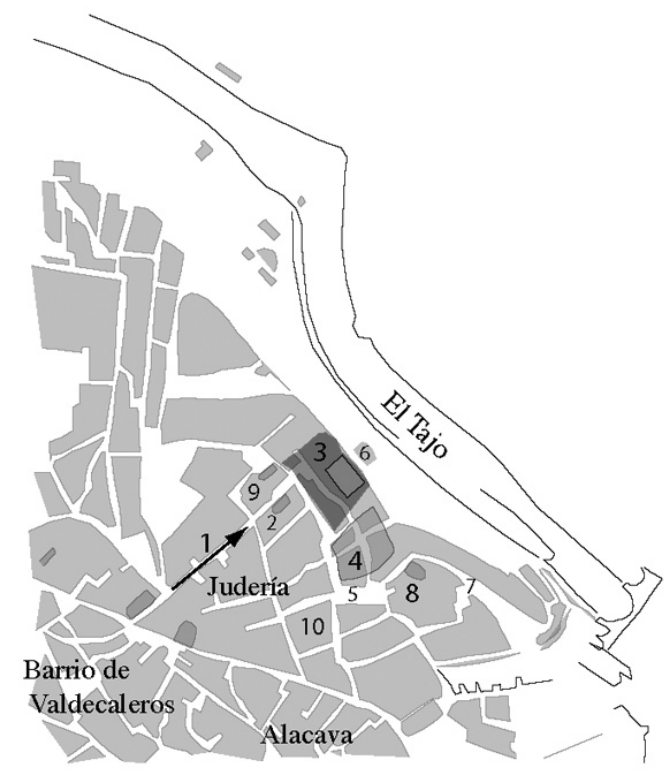

Fig. 5. Plano de El Greco: la manzana que ocupaba el antiguo castillo.

1. Calle del Horno, actual calle de San Juan de Dios. 2. Hospital del Corpus Christi (San Juan de Dios). 3. Manzana de la sinagoga vieja y de la carnicería de los Judíos. 4. Castillo viejo. 5. Plaza del Barrio nuevo. 6. Sitio del amarradero (matadero de las vacas). 7. Cuesta al Tajo. 8. Convento de Santa Ana. 9. Iglesia de San Benito (sinagoga del Tránsito). 10. Iglesia de Santa María la Blanca.

Nosotros hemos podido localizar e identificar una de ellas. Se trata de la del maestro Alejo Venegas, que en $1550^{46}$-la ocupaba desde 1514- vivía en una

${ }^{45}$ En el plano de El Greco (fig. 5), dentro de la manzana que ocupó antes el castillo viejo, se distinguen claramente las dos calles ortogonales que estructuran el espacio del solar parcelado en el siglo XVI. La calle que une la sinagoga del Tránsito con la plaza de Barrio Nuevo y la que baja hacia el «castillo nuevo» y el puente de San Martín están también claramente señaladas.

46 Testamento del maestro Alexo Vanegas del Busto, 15 de mayo de 1550, Toledo. En su primer testamento, de 1550, el maestro Venegas detalla las mejoras que ha ido haciendo en su casa desde 1514: «[...] tengo en Toledo a Barionuevo en la colacion de Sto. Tome; yo he mejorado todo el mueble que en mi casa se hallare; [...] digo que entre los bienes multiplicados es un egonce [sic] de solar que yo cerque delante de la puerta del cerco antiguo que es el sotano de mi casa, el cual solar parecia pertenecia a la dicha puerta, que de verdad es muy antigua, que pienso que ha mas de mil años que es puerta, porque el solar de mi casa se decia el castillo de Barrionuevo, digo digo que yo 
casa cuyo solar «se decia el castillo de Barrio nuevo». A esta casa le había añadido un terreno pequeño al que daba la puerta de atrás, puerta que, practicada en una antigua cerca, abría hacia la parte del río. La descripción de las obras emprendidas por Alejo Venegas y el análisis de la construcción, que se mantiene aún a principios del siglo XXI, nos llevan a identificar su casa con la $\mathrm{n}^{\mathbf{o}} 3$ de la cuesta de Santa Ana, en lo alto de ésta.

En resumen, el estudio de los documentos que mencionan el «castillo viejo» permite precisar la situación de éste: dominando el Tajo, no lejos de la iglesia de Santa María la Blanca, teniendo al este la carnicería, al oeste «la cuesta que desciende del dicho castillo al rio» ${ }^{47}$, frente al futuro convento de Santa Ana. Todo esto enriquece las conclusiones formuladas anteriormente a propósito de la localización de la carnicería y de la sinagoga vieja. El plano de Coello ${ }^{48}$ muestra claramente, a nivel del espacio ocupado por el antiguo castillo de los judíos, en primer lugar la existencia de tres excrecencias geométricas, dos a nivel de la cuesta de Santa Ana, la tercera a la calle de Barrio Nuevo; después, el saliente de la casa de Venegas, sobre el rodadero del Tajo ${ }^{49}$; finalmente, a nivel del espacio precedente y del de la carnicería, el trazado de un camino de ronda (fig. 3).

Sobre el plano catastral actual, el límite del espacio ocupado antiguamente por el «castillo de los judios» se materializa todavía, en primer lugar, en un tramo fuera de alineación en las casas de Barrio Nuevo; en segundo lugar, en la esquina de la calle de San Juan de los Reyes y de la cuesta de Santa Ana, frente a la Escuela de Artes. La esquina del antiguo castillo la ocupaba en la segunda mitad del siglo XX una casa de planta poligonal que presentaba una diferencia de nivel entre dos de sus lados, que se salvaba con unos cuantos escalones. Esta planta poligonal se corresponde con la de una torre cuyos gruesos muros son los que figuran en el plano de $1995^{50}$. Se puede atribuir con certeza a esta torre el

cerque aquella delantera de la dicha puerta antigua habra mas de veinte año y la mostre a los alarifes de la ciudad que entonces eran y ellos dijeron que bien lo pude hacer y cercar, porque les parecia que aquel esconce era perteneciente o pertenecia de la dicha puerta. [...] una pieza, que tiene la puerta a la calle, pueda vivir y edificar en alto y abrir puerta salga al patio principal de mi casa y servirse de ella y de todo comun de la casa hasta la puerta que sale el rio [...]». Publ. I. Adeva Martín, El Maestro Alejo Venegas del Busto, su vida y sus obras (Toledo 1987), págs. 492-506.

${ }^{47}$ León Tello, Judíos de Toledo, t. I, doc. 97, págs. 616-620.

${ }^{48}$ Plano de Francisco Coello, publicado con la colaboración del arquitecto Maximiliano Hijón, 1858, en J. Porres, Plano de Toledo (Toledo 1989).

${ }^{49}$ La casa del maestro Venegas, que privatizó un espacio situado fuera del castillo, se puede ver en una fotografía de principios del siglo XIX.

${ }^{50}$ Expediente Cultural 02091 (M. Lourdes Fernández Gallego, 1995). 
grueso muro aparecido en el curso de las obras de reforma de la antigua casa ${ }^{51}$. Además, en 2003 las excavaciones arqueológicas ${ }^{52}$ hallaron, en el subsuelo de la calle que separaba el castillo de la manzana de la carnicería ${ }^{53}$, las bases de un muro muy grueso y de dos contrafuertes, reveladores de lo que había sido la cerca del castillo de los judíos ${ }^{54}$.

\section{Conclusión}

Seguir los avatares de la «sinagoga vieja», de la «carnicería de los judios» y del «castillo viejo de los judios» más allá del siglo XV, confrontando los textos de que disponemos hasta hoy día y la topografía urbana, nos permite localizar con precisión estos tres elementos de la aljama judía de Toledo (fig. 6). Estaban en lo alto de la colina ocupada por la judería en el siglo XIII, entre la muralla de la ciudad, la futura calle del Horno, la sinagoga, después convertida en iglesia de Santa María la Blanca, y la calle que baja al puente de San Martín. Asimismo hemos localizado el barrio de Arriasa gracias a uno de sus elementos principales, la carnicería, y el castillo en el que terminaba la «calle del barrio de Arriasa».

Este estudio despeja además la duda sobre si hay que atribuir el nombre «sinagoga vieja» a la sinagoga de «Almaliquín», o bien a la que sería iglesia de Santa María la Blanca. Muestra obsoleta la localización de la sinagoga vieja en las inmediaciones del degolladero. Reservamos para un artículo de próxima publicación el análisis detallado de los argumentos que sustentan esta confusión: nos limitaremos por el momento a subrayar que nuestro estudio confirma que los nombres «al degolladero» y «a la juderia» designan dos barrios distintos ${ }^{55}$.

${ }^{51}$ Casa $\mathrm{n}^{\circ} 13$, calle de los Reyes Católicos. La casa distaba varios metros del emplazamiento del pozo público llamado «de Barrio Nuevo», todavía presente a principios del último cuarto del siglo xx. Esta casa ha sido reemplazada por una tienda que mantiene la misma planta poligonal, pero no la escalera de acceso a la vivienda.

${ }^{52}$ Queremos agradecer a Elena Sánchez el habernos facilitado la consulta y utilización de los resultados de las excavaciones que tuvo a su cargo: E. I. SÁnchEz PELÁEz, Proyecto de ejecución de rehabilitación de edificio para hotel de cuatro estrellas en calle Reyes Católicos, $n^{\circ} 5$ (Toledo 2002).

53 La calle fue privatizada y sobre ella se construyó en los últimos años del siglo XX.

${ }^{54}$ La prolongación de este muro, que llegaba a la muralla de la ciudad, y cuyos restos están incluidos hoy en el interior del edificio de la Real Fundación de Toledo, es visible en fotos aéreas de 1945.

55 Barrios mencionados en los libros de «rentas del refitor», redactados en 1354 (cit. LEÓN Tello, Judíos de Toledo, t. II, doc. 496, pág. 135), 1372 (ibid. doc. 571, pág. 159), y en 1394-1395 (ibid., doc. 634, págs. 183-184). 


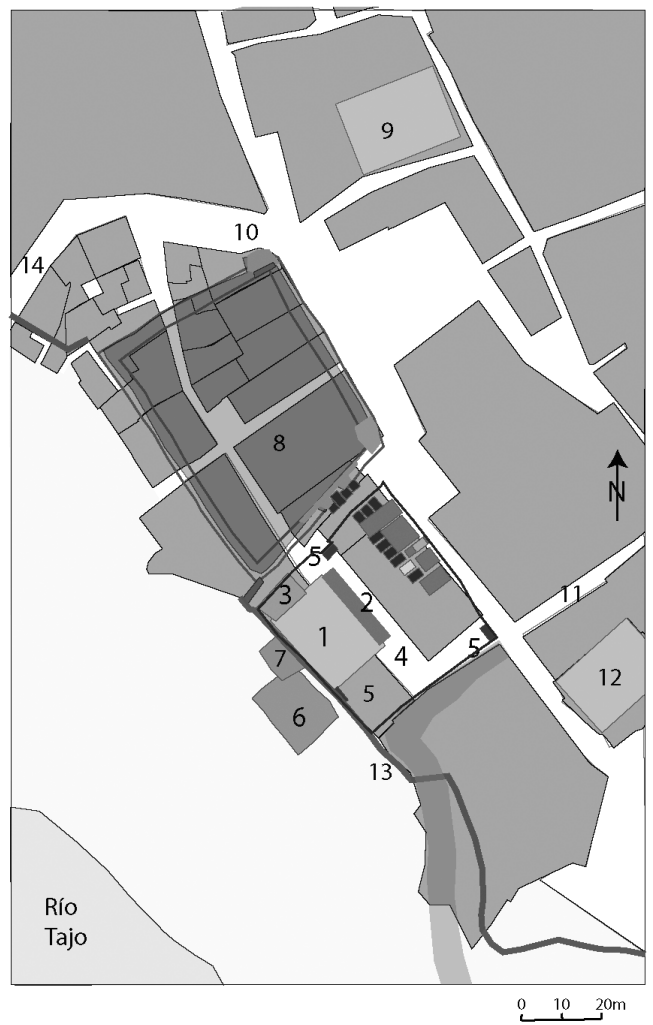

Fig. 6. Restitución de la carnicería, de la sinagoga vieja y del castillo en el siglo XV.

1. Sinagoga Vieja. 2. Portal de los carniceros. 3. Hospital de la aljama. 4. Carnicería de los Judíos. 5. Entrada de las carnicerías de los judíos. 6. Corral conocido como «amarradero de las vacas». 7. Huerta pequeña y covachuelas. 8. Castillo de los judíos 9. Sinagoga Sta. María la Blanca. 10. Plaza de Barrio nuevo 11. Calle de la Judería, hoy San Juan de Dios. 12. Sinagoga del Tránsito. 13. Muro de la ciudad. 14. Cuesta hacia el puente de San Martín.

Si bien completa nuestros trabajos precedentes ${ }^{56} \mathrm{y}$ perfila con mayor precisión el diseño de la Judería Mayor, somos conscientes de que el presente estudio

${ }^{56}$ Ver J. PAssinI, «La juiverie de Tolède: bains et impasses du quartier de Hamanzeit», en El legado material hispanojudío. VII Curso de Cultura Hispanojudía y Sefardí, A. M. López Álvarez y R. IzQuierdo Benito, coords. (Cuenca 1997), págs. 301-326; IDEM, «La sinagoga del Sofer en Toledo», Sef 64 (2004), págs. 141-157; e IDEM, «La sinagoga del barrio de Caleros», Sef 66 (2006), págs. 69-88. 
deja numerosas cuestiones sin solventar. Por otra parte, pone de relieve la necesidad urgente de nuevas y detalladas prospecciones arqueológicas que vayan precedidas de nuevos y detallados estudios de los textos medievales.

Recibido: 16/01/2007

Aceptado: 20/10/2007 
